2021, Band 9, Heft 1/2

Seiten 185-191

zeitschrift-suburban.de

10.36900/suburban.v9i1/2.678

\title{
Höher, weiter, breiter. Die endlose Stadt nach Covid-19
}

Kommentar zu Stefan Höhne und Boris Michel „Das Ende des Städtischen?

Pandemie, Digitalisierung und planetarische Enturbanisierung“

Stefan Höhne, Boris Michel ,Das Ende des Städtischen? Pandemie, Digitalisierung und planetarische Enturbanisierung'

Kommentare von:

Marcelo Lopes de Souza, Matthias Naumann, Anke Strüver, Markus Kip, Hannah Schilling, Roger Keil, Christian Haid, Anna-Lisa Müller, Nikolai Roskamm

Roger Keil

„Nach der Pandemie ist vor der Pandemie!“

(Volksmund, frei nach Sepp Herberger, deutscher Fußballtrainer)

Der inspirierende Debattenaufschlag von Stefan Höhne und Boris Michel (2021) lädt uns ein, über die Zukunft des Städtischen nach der Covid19-Pandemie nachzudenken.[1] Die neun Symptome einer Krise der Stadt sind aber unter Umständen nur konzeptionell zu fassen, nicht als solche erfahrbar. Leben die Stadtbewohner_innen und -nutzer_innen bewusst als solche in der vollständig „verstädterten Gesellschaft“ (Lefebvre 1972: 7) und erleben sie deren neunköpfige Krise als Krise der Stadt? Oder werden die Krisenerscheinungen des Städtischen vor, in und nach der Pandemie in anderen Registern erfahren und abgebildet: als Krise des Gesundheitswesens, als Krise der Altenpflege, oder als Krise der Pflege insgesamt, als Krise der Einwanderungsgesellschaft, als Krise des racial capitalism, als Krise der Arbeitsbedingungen in der Pflege und den essential services generell, die Krise von Politik und Governance, die Krise der Demokratie, und so fort? Diese Fragen sind natürlich im Rahmen des vorliegenden Kommentars nur als rhetorische zu behandeln. Sie lassen sich nicht zufriedenstellend beantworten und die Autoren weisen ja bereits selbst auf „die Grenzen einer allein auf Stadt und Urbanität abhebenden Krisendiagnostik“ hin (Höhne/Michel 2021: 146). Ich werde es also auf den wenigen Seiten, die mir hier zur Verfügung stehen, gar nicht erst versuchen, sondern in drei Teilen die Beziehungen des Städtischen und der Stadtforschung zum Thema Infektionskrankheiten am aktuellen Beispiel der Coronaviruspandemie andiskutieren. Meine Gedanken zu diesen Überlagerungen beginnen mit der Periode vor der Pandemie, behandeln die Stadt(-forschung und -praxis) während der Pandemie und führen schließlich über den - derzeit noch hypothetischen - Punkt hinaus, an dem die Covid-19-Krise überwunden sein wird. 


\section{Vor der Pandemie}

Das war vorauszusehen. Nichts von dem, was wir in den letzten zwölf Monaten weltweit erlebt haben, kam unerwartet. Unter dem Eindruck der sich ausbreitenden Urbanisierung hatten seit Jahrzehnten Epidemiolog_innen und Expert_innen des öffentlichen Gesundheitswesens weltweit vor der ansteigenden Gefahr einer massiven Epidemie gewarnt: Ausgehend von den Rändern der globalen massiven Verstädterung würden sich die entstehenden Infektionskrankheiten vornehmlich in den Städten ihre Opfer suchen. Aber die zunehmende Urbanisierung wurde auch als Chance gewertet, die Krankheitsbekämpfung zu verbessern (Bollyky 2018a, 2018b). Die Erweiterung des globalen Städtesystems selbst, vor allem das Netzwerk der global cities, war schon beim Ausbruch des Severe Acute Respiratory Syndrome (SARS) im Jahre 2003, das vor allem große Finanzzentren wie Hongkong, Singapur und Toronto heimsuchte, als Faktor des Ursprungs, der Verbreitung und letztlich der Überwindung der Infektionskrankheit diskutiert worden (Ali/Keil 2008). Nicht viel später, in den Jahren 2014/15, folgte die westafrikanische Ebolaepidemie, die vor allem die Länder entlang des Manoflusses, Guinea, Sierra Leone und Liberia, traf. Ebola virus disease, vormals nur in entfernten ländlichen Gebieten Zentralafrikas zu Hause, trat hier jedoch erstmals als städtisches Phänomen auf und führte zu hohen Ansteckungszahlen in den informellen Siedlungen der Städte Monrovia und Freetown, aber auch zu innovativen zivilgesellschaftlichen Initiativen zur Bekämpfung der Krankheit, die auch heute im Kampf gegen die Coronapandemie zur Anwendung kommen (Ali et al. i. E.).

Während SARS 2003 das Exoskelett des globalen Kapitalismus infizierte, dringt heute Covid-19 zum Fleisch und Blut des planetarischen Urbanismus vor. Die Kulturanthropologin Meike Wolf hat bereits vor einigen Jahren auf die Verbindung von Verstädterung und entstehenden Infektionskrankheiten hingewiesen, jedoch davor gewarnt, beide Prozesse als natürlich misszuverstehen (2016: 961). Wenn also weder „Städte selbstverständliche und universelle Formationen sind und die Entstehung von Krankheiten kein ,natürliches ' Ereignis“" ist (ebd.), dann erfordert es andauernde und tiefgehende Forschung, um ihr Verhältnis unter den sich ständig ändernden Bedingungen zu beleuchten und letztlich zu verstehen.

In diesem Sinne haben meine Kollegen Creighton Connolly, S. Harris Ali und ich über die letzten Jahre versucht, die spezifischen städtischen und politischen Pathologien der „vollständig verstädterten Gesellschaft“ vor allem aus der Perspektive der globalen Suburbanisierung zu verstehen (Connolly/ Keil/Ali 2020). Diese strategische Verschiebung der Perspektive von der Krankheit in der Stadt zur Krankheit im Städtischen ist aus unserer Sicht folgenreich. Mit der Betonung der systematischen Natur der Beziehungen von Stadt und Krankheit in der Periode der extensiven Urbanisierung nehmen wir Abschied von der traditionellen ortsspezifischen Analyse (ein bestimmter Stadtteil, eine bestimmte Krankheit), die die Forschung seit dem 19. Jahrhundert begleitete, und lassen auch die Betonung nur ausgewählter Städte hinter uns (global cities). Stattdessen argumentieren wir, dass das Verhältnis von Verstädterung und entstehenden Infektionskrankheiten weltweit durch demografische, infrastrukturelle und Governance-bezogene 
Veränderungen in Bewegung gekommen ist. Dies ist nicht mit der These zu verwechseln, nach der Dichtegradienten direkt mit der Verbreitung von Krankheiten korrelieren.[2] Die extensive Urbanisierung bedeutet einfach, dass das städtische Gefüge höher, weiter und breiter wird und dass die endlose Stadt nun als Kontext für die Entwicklung, Verbreitung und letztlich für die Überwindung der Krankheiten gesehen werden muss.

\section{Während der Pandemie}

Diesen Gedanken nahmen wir zu Beginn der Covid-19-Pandemie auf und stellten die Hypothese auf, dass Covid-19 an den Peripherien der verstädterten Gesellschaft andockt (Biglieri/De Vidovich/Keil 2020). Ich will dieses auch im Debattenaufschlag angesprochene Argument hier weder vertiefen noch erneut aufrollen, sondern eine spezifische Denkweise diskutieren, die sich aus dieser Einsicht ergibt. Zunächst war und ist damit nicht gemeint, dass das Coronavirus eine Vorliebe für die suburbs hat, obwohl das durchaus Teil des Narrativs sein könnte. Vielmehr ist hier angesprochen, dass das Virus seine Wirksamkeit an den räumlichen, gesellschaftlichen und institutionellen Rändern der städtischen Gesellschaft entfaltet und dort einen Großteil seiner Opfer sucht. In Übereinstimmung mit Meike Wolfs (2016) Warnung, dass sich weder das Städtische noch der Erreger, natürlich verhalten und offenbaren, ist hier festzuhalten, dass die periphere Position des Virus Sars-CoV-2 und die Krankheit, die es auslöst - Covid-19 -, nicht einfach nur von der sozialstatistischen Karte einer Stadt oder einer Region ablesbar sein wird. Stattdessen haben wir während der Pandemie gelernt, dass mit jeder neuen Einsicht in das Virus und mit jeder neuen Einwirkung der Krankheit auf die menschlichen Gemeinschaften in unseren Städten ein neuer Typus von Erreger (mit neuen Eigenschaften) entsteht und mit jeder neuen gesellschaftlichen Interaktion mit dem Pathogen neue städtische Realitäten geschaffen wurden. Das bedeutet auch, dass das Virus nicht einfach vorher verschleierte Wahrheiten über unsere Städte, enthüllt', vielmehr nimmt es an der Gestaltung dieser Wahrheiten selbst aktiv teil.

Nehmen wir die anscheinend simple sozialwissenschaftliche Tatsache zum Ausgangspunkt, dass Menschen, die sich in sozioökonomisch schwacher Position befinden, oder noch ausgeprägter: die in den Teilen der Stadt leben, wo diese wirtschaftlich benachteiligten Personen leben, dem Virus gegenüber anfälliger sind als Menschen, die wirtschaftlich abgesichert sind. Statistisch ist das wahrscheinlich und plausibel. Doch die periphere Position dieser Menschen ist keine fixe quantitative Realität, sondern ein wandelbares Produkt schnell wechselnder Einflüsse und langwieriger Prozesse sozialer Unterdrückung. So ist beispielsweise einsichtig, dass viele arme Menschen nicht-weißer Herkunft sind, oft Migrant_innen und häufig Mieter_innen an den Rändern des Wohnungs- und Arbeitsmarktes sind. Zudem sind sie auf das öffentliche Verkehrssystem der Stadt alternativlos angewiesen und leben weiter vom Arbeitsplatz entfernt, vor allem diejenigen, die zwei oder mehr prekäre Arbeitsplätze haben, um über die Runden zu kommen. Damit haben sich unsere einfachen statistischen Wahrheiten der ursprünglichen Annahme, dass sich das Virus die Ränder der Gesellschaft sucht, bereits ausgiebig verkompliziert. 
Doch nicht nur das Bild der Randständigkeit ist hier komplexer geworden. Auch sind die Kategorien, in denen sich die periphere Natur des Städtischen erkennen lässt, von Land zu Land und von Stadt zu Stadt verschieden. Was genau schwarze Hautfarbe auf dem Arbeits- oder Wohnungsmarkt bedeutet, kann durchaus unterschiedlich sein, wenn wir zum Beispiel Berlin, Milano oder Toronto in Betracht ziehen. Schwarze Menschen werden in diesen drei Städten von den Behörden nicht in identischer Weise erfasst. Hier ist nicht der Ort, die geschichtlichen Gründe für diese Unterschiede im Einzelnen zu erläutern. Doch es ist bezeichnend und sollte für sich sprechen, dass in Toronto während der Coronakrise die ursprüngliche Nichterfassung schwarzer Menschen in den Statistiken des öffentlichen Gesundheitswesens zum Politikum geworden ist und daraufhin geändert wurde. Wir wissen jetzt, wo genau in der Stadtregion nicht-weiße Menschen von der Pandemie betroffen sind und wer ihr zum Opfer fällt. Vor allem die eng gestaffelten Hochhausviertel in den inneren Vorstädten und die ausufernden ethnoburbs in der weiteren Peripherie der Region, seit Langem mehrheitlich von Migrant_innen, oft prekär untergebrachten Mieter_innen[3] und Menschen nicht-weißer Hautfarbe bewohnt, stechen in den derart nuancierten Statistiken klar hervor. Sie werden bewohnt von denen, die nicht zu Hause arbeiten können und die Stadtregion täglich dichtgedrängt in Bussen durchqueren, um zu ihren Arbeitsplätzen in schlecht bezahlten Dienstleistungs-, Lager-, Logistik- oder Fabrikjobs zu gelangen. Dadurch werden dramatische Ungleichheiten offenbar, die über die rein statistischen Informationen hinausdeuten und komplexe Zusammenhänge zwischen Grundbesitz, Bildungs- und Berufschancen und dem Gesundheitssystem offenlegen.

Wenn diese relevanten Bezüge nicht bekannt sind, fehlt die Grundlage für einen vernünftigen Diskurs über die Muster der sozialräumlichen Differenzierung. Selbstverständlich bergen die möglichen Schäden einer Markierung rassischer oder ethnischer Unterschiede die Gefahr der Stigmatisierung bestimmter Familien, Stadtteile oder Communities, der durch eine bewusste Gegensteuerung begegnet werden muss. Aber wenn sich die massiv ausdifferenzierte städtische Gesellschaft in der Krise kein Bild von sich machen kann, wird sie die drastischen Ungleichheiten nicht ausmerzen können, die durch die Krise unverkennbar geworden sind.

Doch gehen wir noch einen Schritt weiter. Die sozialen, räumlichen und institutionellen Peripherien in der Pandemie wurden wie gezeigt mit neuer - oft trauriger und deprimierender - Bedeutung angereichert. Doch die von institutioneller rassistischer und sozialer Diskriminierung betroffenen Subjekte erlebten im Sommer 2020 in der Bugwelle des ersten Infektionsschubs einen politisch-revolutionären Schub, der die Wirkung der Anerkennung ihrer statistischen Existenz bei Weitem an Bedeutung übertraf. Ich spreche hier natürlich von der Ermordung des Afroamerikaners George Floyd durch einen Polizeibeamten in Minneapolis im Mai des Jahres. Die Protestwelle, die von den Städten der USA um die Welt schwappte, radikalisierte die seit Jahren präsente Bewegung gegen Polizeigewalt und prangerte den institutionellen und alltäglichen Rassismus gegen schwarze Menschen weltweit an. Aus dieser Situation wurden die Grundlagen geschaffen, einerseits schrittweise Reformen im Gesundheitswesen, in der Mietrechtspraxis (zum Schutz von Menschen, die von Räumung bedroht sind) und in den 
Beschäftigungsverhältnissen in Niedriglohnsektoren durchzusetzen, die durch Covid-19 angeregt wurden. Andererseits wurden die Voraussetzungen dafür gesetzt, dass die betroffenen Menschen selbst zu handelnden Subjekten werden, deren Forderungen über die Reformen hinausweisen. Die Geschichte wird zeigen, wie viele der durchaus revolutionären Forderungen der Black Lives Matter Bewegung - wie etwa die, der Polizei die Finanzierung zu entziehen - ,greifen“ und zu dauerhaften Verbesserungen führen werden (wie z. B. die radikalen bürgerrechtlichen Reformen der 1960er Jahre).

Doch steht außer Zweifel, dass die afroamerikanische Befreiungsbewegung die städtischen Verhältnisse gerade dann zum Tanzen brachte, als das Virus drohte, in den nicht-weißen Quartieren, unter den schlechtbezahltesten Beschäftigten und den am Wohnungsmarkt ausgegrenzten Menschen nichtweißer Hautfarbe den schlimmsten Schaden anzurichten. Die Wirkung dieser Politisierung kann nicht überbewertet werden. Und dann trifft auch das zu: Wenn auch in Toronto, Berlin und Milano, um bei diesen Beispielen zu bleiben, sich Menschen nicht-weißer Hautfarbe in spürbar unterschiedlichen städtischen Realitäten an unterschiedlichen Peripherien wiederfanden, so waren sie schließlich durch die globalen Aufstände in der Folge des Mordes an George Floyd vereint.

Als Fazit oder Fußnote muss ich an dieser Stelle anmerken, dass der schlimmste Aspekt des Querdenkertums und der Coronaleugnung darin besteht, dass diese komplexen Prozesse der Exposition sozialer Ungerechtigkeiten schlichtweg übersehen, vielleicht auch bewusst ausgeblendet werden, was die Humanität derjenigen Stadtbewohner_innen auslöscht, die am härtesten von der Pandemie betroffen sind.[4]

\section{Nach der Pandemie}

Wie geht es von hier aus weiter? Das kann keine Frage der unbegründeten Zukunftsdeutung sein. Wir konnten im letzten Jahr beobachten, wie uns die Entwicklung von Covid-19 eine städtische Gesellschaft präsentierte, in der die Stadt und das Virus selbst nicht statisch blieben, sondern ständig neu definiert wurden und in keiner Weise ,natürlich` zueinander passten. Und mit Blick auf die politische Aufstandsbewegung Black Lives Matter, die die Grenzen der gesellschaftlichen Möglichkeiten (und Verpflichtungen) inmitten einer lebensbedrohlichen Krise neu zog, wurde klar, dass die latenten Verwerfungen, die den extensiven Urbanismus heute ausmachen, nun manifest geworden sind. Eine Forschungsgruppe, der ich angehöre (Allahwala et al., i. E.), findet in einem politökonomisch begründeten Vergleich der gesellschaftlichen Reaktionen in Berlin und Toronto, dass diese städtischen Gesellschaften einen demokratischen Moment erleben, der die gegebenen Verhältnisse städtischen Lebens potenziell aus dem Gleichgewicht bringen kann. Wir schließen uns hier ähnlichen Argumentationsmustern an, wie wir sie in der Zero-Covid-Bewegung in Europa feststellen können - ein Aufruf zur gesellschaftlichen Solidarität, die eine Rückkehr zu den Verhältnissen vor der Manifestation der Krise unmöglich zu machen scheint (ZeroCovid 2020).

Die Forderungen nach bleibenden strukturellen Verbesserungen der Lebensverhältnisse derjenigen in unserer städtischen Gesellschaft, die am meisten unter der Pandemie leiden müssen, sind nicht mehr zu übersehen. 
Konkret heißt das, dass die Herausforderungen, die von der grundsätzlichen und systematischen sozialen, räumlichen und institutionellen Marginalisierung von Teilen der städtischen Gesellschaft herrühren, nach der Pandemie nur unter schweren Kosten ignoriert werden können. Sicher, die Gefahren liegen auf der Hand - wie auch Höhne und Michel in ihrem Debattenaufschlag deutlich vor Augen führen: Sie liegen vor allem im schier unbegrenzten Potenzial der gekoppelten Macht von Konsum und Überwachung, die uns die digitalen Großkonzerne in der Krise vorführen, in den falschen Versprechungen der, intelligenten Stadt' und im Vergessen und der Unterdrückung des, demokratischen Moments'. In der Verarbeitung dieser Pandemie finden sich grundlegende Hinweise auf die Prinzipien des Handelns, die beim nächsten Krisenszenario - Klimawandel, Verelendung, Krieg, Flucht, und auch wieder Pandemie - in unserer städtischen Gesellschaft zur Anwendung gebracht werden müssen (Tzaninis et al. 2020). Unterdessen werden die Städte im Prozess der extensiven Urbanisierung weiterhin höher, breiter und weiter in Struktur und Reichweite.

In diesem Sinne - und mit Bitte um Verzeihung an den alten Fußballtrainer - müssen radikale Stadtforscher_innen und -aktivist_innen bereit sein, ihre Augen schon jetzt von der Reaktion auf Covid-19 in Richtung der nächsten Bedrohung zu lenken. Die nun angelaufenen Impfungen werden viele Menschenleben retten und es uns erlauben, die Städte in den nächsten Jahren wiederum dem Leben zu öffnen, doch die Vakzine sind nicht geeignet, die städtische Gesellschaft gegen Ungleichheit und Ungerechtigkeit zu impfen, die das Elend und den Tod an den gesellschaftlichen, räumlichen und institutionellen Peripherien der städtischen Gesellschaft zu verantworten haben. Daran können nur die Städter_innen selbst etwas ändern. Diese Aufgabe liegt nun klar vor uns.

Dieser Artikel wurde durch Mittel der York University Toronto gefördert.

\section{Endnoten}

[1] Danke an Ahmed Allahwala und Sabine Bröck fürs Querlesen und an die Redaktion von $\mathrm{s} \mathrm{u} \mathrm{b} \backslash \mathrm{u}$ r b a n für die sehr willkommenen Anregungen zur Verbesserung des Textes (insbesondere in Bezug auf mein ,Denglisch'). Der strukturierende Kunstgriff von vor, während und nach ist nicht als rein chronologisch misszuverstehen.

[2] Das haben auch andere bereits für die gegenwärtige Covid-19-Krise gezeigt: Boterman 2020; Keil 2021; Moos/McCulley/Vinodrai 2020.

[3] Aus vielen kulturellen und sozioökonomischen Gründen, die hier nicht weiter erörtert werden können, spielt Grundbesitz, und damit die Frage Hauseigentum oder Wohnungsmiete, in der Siedlerkolonie Kanada eine andere Rolle als in vielen europäischen Ländern, vor allem in Deutschland. Generell bleibt das Hauseigentum erstrebenswert und wird oft mit einem Mittelklassestatus gleichgesetzt. Tatsächlich lebt mehr als die Hälfte der Bevölkerung Torontos zur Miete, oft in der Situation ständiger finanzieller Bedrohung aufgrund hoher Mietpreise. Vor der Pandemie hatte eine einschlägige Studie ergeben, dass 46 Prozent der zur Miete wohnenden Haushalte in Toronto mehr als 30 Prozent ihres Einkommens für Wohnen ausgeben, was allgemein als Massstabsgrenze für die Bezahlbarkeit von Wohnraum angenommen wird (Mathieu 2018).

[4] In einem stimulierenden Beitrag zog Ingar Solty (2020) bereits im Herbst 2020 eine traurige Bilanz des aufmüpfigen Querdenkertums, der Verschwörungstheoretiker_innen und „Covidioten“, dem ich hier nichts hinzufügen möchte. 


\section{Autor_innen}

Roger Keil ist Stadt- und Umweltwissenschaftler und forscht zu globaler Suburbanisierung, städtischer politischer Ökologie, Stadt und Infektionskrankheiten und regionaler Governance. rkeil@yorku.ca

\section{Literatur}

Ali, S. Harris / Keil, Roger (Hg.) (2008): Networked disease. Emerging infections in the global city. Oxford: Wiley-Blackwell.

Ali, S. Harris / Fallah, Mosoka / McCarthy, Joseph / Keil, Roger / Connolly, Creighton (i. E.): Mobilizing the urban social infrastructure of informal settlements in infectious disease response - the case of ebola virus disease and COVID-19 in West Africa. In: Landscape and Urban Planning.

Allahwala, Ahmed / Carvalho, Nigel / Keil, Roger / Ritch, Jenna (i. E.): Confronting COVID-19 in collaboration and conflict. The political economies of federal pandemic responses in Canada and Germany. In: Studies in Political Economy.

Biglieri, Samantha/ De Vidovich, Lorenzo / Keil, Roger (2020): City as the core of contagion? Repositioning COVID-19 at the social and spatial periphery of urban society. In: Cities \& Health. https://doi.org/10.1080/23748834.2020.1788320.

Bollyky, Thomas J. (2018a): Plagues and the paradox of progress. Why the world is getting healthier in worrisome ways. Cambridge, MA: The MIT Press.

Bollyky, Thomas J. (2018b): The future of global health is urban health. In: Council on Foreign Relations, 29.11.2018. https://www.cfr.org/article/future-global-health-urban-health.

Boterman, Willem R. (2020): Urban-rural polarisation in times of the corona outbreak? The early demographic and geographic patterns of the SARS-CoV-2 epidemic in the Netherlands. In: Tijdschrift voor Economische en Sociale Geografie 111/3: 513-529.

Connolly, Creighton / Keil, Roger / Ali, S. Harris (2020): Extended urbanisation and the spatialities of infectious disease: Demographic change, infrastructure and governance. In: Urban Studies. https://doi.org/10.1177/0042098020910873.

Höhne, Stefan / Michel, Boris (2021): Das Ende des Städtischen? Pandemie, Digitalisierung und planetarische Enturbanisierung. In: sub \urban. zeitschrift für kritische stadtforschung 9/1-2, 141-149.

Keil, Roger (2021): The density dilemma. There is always too much and too little of it. In: Urban Geography 41/10, 1284-1293.

Lefebvre, Henri (1972): Die Revolution der Städte. München: List Verlag.

Mathieu, Emily (2018): Almost half of Toronto tenants paying „unaffordable“ rent, study finds. In: Toronto Star, 22.5.2018. https://www.thestar.com/news/gta/2018/05/22/ almost-half-of-toronto-tenants-paying-unaffordable-rent-study-finds.html (letzter Zugriff am 22.2.2021).

Moos, Markus / McCulley, Amanda / Vinodrai, Tara (2020): COVID-19 and urban density: Evaluating the arguments, discussion report. University of Waterloo. https:// uwaterloo.ca/environment/sites/ca.environment/files/uploads/files/densityhousing_1_ arguments_moosmcculleyvinodrai.pdf (letzter Zugriff am 22.2.2021).

Solty, Ingar (2020): Wer glaubt warum an Verschwörungstheorien? Ein Versuch zu verstehen. In: Luxemburg Online, Oktober 2020. https://www.zeitschrift-luxemburg.de/ wer-glaubt-warum-an-verschwoerungstheorienein-versuch-zu-verstehen (letzter Zugriff am 22.2.2021).

Tzaninis, Yannis / Mandler, Tait / Kaika, Maria / Keil, Roger (2020): Moving urban political ecology beyond the „urbanization of nature“. In: Progress in Human Geography. https:// doi.org/10.1177/0309132520903350.

Wolf, Meike (2016): Rethinking urban epidemiology. Natures, networks and materialities. In: International Journal of Urban and Regional Research 40/5: 958-982.

ZeroCovid (2020): \#ZeroCovid: The aim is zero infections! For a European shutdown in solidarity. https://zero-covid.org/language/en/ (letzter Zugriff am 18.1.2021). 
